\title{
NUMERICAL INTEGRATION RULES FOR MULTIVARIATE INVERSIONS
}

\author{
N. G. SHEPHARD \\ Department of Statistics, London School of Economics, London WC2A $2 A E, U K$
}

(Received 26 July 1990; in final form 4 December 1990)

\begin{abstract}
Working from a known characteristic function, integration rules for the computation of the multivariate distribution function are derived. Procedures for the automatic selection of step sizes are one particular strength of the proposed method. Examples of the use of the procedure are given.
\end{abstract}

KEY WORDS: Characteristic functions, numerical integration, inversion theorems, Imhof procedure, correlation coefficients

\section{INTRODUCTION}

In this paper we develop rules for the numerical inversion of multivariate characteristic functions in order to compute the distribution function. As such they represent multivariate generalisations of the Imhof (1961) and Davies $(1973,1980)$ techiques.

An example of the use of the multivariate inversion algorithm is discussed at length, while three other cases of its use are referenced.

\section{THE UNIVARIATE CASE}

We will suppose $\phi$ is a known characteristic function corresponding to the distribution function $F$ which in turn possesses a density $f$. For ease of exposition we will assume $f$ and $\phi$ are Lebesgue integrable and that the random variable of interest possesses a mean. These last three assumptions can be dropped without affecting our integration rules by using principle values of Lebesgue integrals, but we find that these types of details detract from the logical structure of our approach and so prefer to maintain our stated assumptions. These will allow us to develop the theory using standard manipulative techniques.

Gurland (1948) showed that $F$ and $\phi$ are related in the following way

$$
F(x)=\frac{1}{2}-\frac{1}{2 \pi} \int_{0}^{\infty}\left(\Delta_{t}\left[\frac{\phi(t) e^{-i t x}}{i t}\right]\right) d t
$$

where $\Delta_{t} \eta(t)=\eta(t)+\eta(-t)$. 
Following Davies (1973) we will rewrite this to give an expression which can be used to numerically invert characteristic functions. However, our proof is somewhat different from that used by Davies. Throughout the remaining parts of this paper we will exploit the operator $\delta(g, j)$ which is defined so that $\delta(g, j) \operatorname{Pr}(X \leqq x)=$ $\operatorname{Pr}\left(X \leqq x-\left(2 \pi j / h_{g}\right)\right)-\operatorname{Pr}\left(X>x+\left(2 \pi j / h_{g}\right)\right)$. In what follows $h_{g}$ should be thought of as the step size of the integration procedure.

Theorem 1 (Davies (1973)) For $h_{1}>0$,

$$
\begin{aligned}
F(x) & +\sum_{j=1}^{\infty} \cos [2 \pi z j][\delta(1, j) \operatorname{Pr}(X \leqq x)] \\
= & \frac{1}{2}-\frac{1}{2 \pi} h_{1} \operatorname{Im}\left[\frac{\phi\left(h_{1} z\right) \exp \left[-i x h_{1} z\right]}{h_{1} z}\right] \\
& -\frac{1}{2 \pi} h_{1} \sum_{v=1}^{\infty}\left(\Delta_{z} \operatorname{Im}\left[\frac{\phi\left(h_{1}(z+v)\right) \exp \left[-i x h_{1}(z+v)\right]}{h_{1}(z+v)}\right]\right),
\end{aligned}
$$

Where $\operatorname{Im}(\cdot)$ denotes the imaginary part of a complex function.

Proof Given in the appendix.

The second term of the left-hand side of this expression can be viewed as an error caused by approximating the inversion integral.

Allowing $z$ to be zero means the integration is performed by the trapezium rule. As the induced integration error is

$$
\left|\sum_{j=1}^{\infty}[\delta(1, j) \operatorname{Pr}(X \leqq x)]\right|
$$

which is rather difficult to manipulate, this rule has not been used for this type of inversion. Instead the simpler Riemann sum has been exploited. This occurs when we allow $z=\frac{1}{2}$.

Corollary 1 (Davies (1973)) For $h_{1}>0$,

$$
\begin{aligned}
& F(x)+\sum_{j=1}^{\infty}(-1)^{j}[\delta(1, j) \operatorname{Pr}(X \leqq x)] \\
& =\frac{1}{2}-\frac{1}{\pi} h_{1} \sum_{v=0}^{\infty} \operatorname{Im}\left[\frac{\phi\left(h_{1}\left(v+\frac{1}{2}\right)\right) \exp \left[-i x h_{1}\left(v+\frac{1}{2}\right)\right]}{h_{1}\left(v+\frac{1}{2}\right)}\right] .
\end{aligned}
$$


The induced numerical integration error is much simpler here. The absolute value of the error is,

$$
\left|\sum_{j=1}^{\infty}(-1)^{j}[\delta(1, j) \operatorname{Pr}(X \leqq x)]\right| \leqq \max \left[F\left(x-\frac{2 \pi}{h_{1}}\right), 1-F\left(x+\frac{2 \pi}{h_{1}}\right)\right] .
$$

In turn we can deduce an automatic criterion for the selection of $h_{1}$ so that this error is small. If $\phi$ is analytic then the corresponding moment generating function will exist. We will write it as $M$, then for small $u>0$ we have (see Davies (1973)) $\quad 1-F(x) \leqq M(u) \exp (-u x) . \quad$ Writing $\quad \psi(u)=\log M(u) \quad$ and selecting $x=\psi^{\prime}(u)=d \psi(u) / d u$ implies we have $1-F\left(\psi^{\prime}(u)\right) \leqq \exp \left(-u \cdot \psi^{\prime}(u)+\psi(u)\right)$. Equally $F\left(\psi^{\prime}(u)\right) \leqq \exp \left[\psi(-u)+u \psi^{\prime}(u)\right]$. Therefore, by appropriate choice of $h_{1}$, we can bound this induced error by any small positive real $\varepsilon$.

This formulation has successfully been applied to the problem of finding the exact distribution function of a quadratic form in normal variables by Davies (1980).

\section{THE BIVARIATE CASE}

In this section we transfer the development given in Section 2 over to the case of a bivariate distribution function. From Gurland (1948) or more easily Shephard (1991) we have

$$
\begin{aligned}
u\left(x_{1}, x_{2}\right) & =4 F\left(x_{1}, x_{2}\right)-2\left[F\left(x_{1}\right)+F\left(x_{2}\right)\right]+1 \\
& =\frac{2^{2}}{(2 \pi)^{2}} \int_{0}^{\infty} \int_{0}^{\infty}\left(\Delta_{t_{1}} \cdot \Delta_{t_{2}}\left[\frac{\phi(t) e^{-i x^{\prime} t}}{i t_{1} i t_{2}}\right]\right) d t_{1} d t_{2} .
\end{aligned}
$$

To make the notation in the theorem reasonably compact we will use $\sum_{j=0}^{\infty}$ to denote the double sum over the variables $j_{1}$ and $j_{2}$ which vary between zero and infinity, but never equal zero simultaneously.

THEOREM 2 For $h_{1}, h_{2}>0$

$$
\begin{aligned}
2^{-2} u\left(x_{1}, x_{2}\right) & +\sum_{j=0}^{\infty}\left(\left[\prod_{k=1}^{2} \cos \left[2 \pi z_{k} j_{k}\right] \delta\left(k, j_{k}\right)\right] F\left(x_{1}, x_{2}\right)\right) \\
= & \frac{-\frac{1}{2}}{(2 \pi)^{2}} h_{1} h_{2}\left[\sum_{v_{2}=-\infty}^{\infty}\left(\Delta_{z_{2}} \operatorname{Re}\left[\frac{\phi\left(h_{1} z_{1}, a_{2}\right) e^{-i x^{\prime}\left(h_{1} z_{1}, a_{2}\right)^{\prime}}}{h_{1} z_{1} a_{2}}\right]\right)\right. \\
& \left.+\sum_{v_{1}=1}^{\infty} \sum_{v_{2}=-\infty}^{\infty}\left(\Delta_{z_{1}} \cdot \Delta_{z_{2}} \operatorname{Re}\left[\frac{\phi(a) e^{-i x^{\prime} a}}{a_{1} a_{2}}\right]\right)\right]
\end{aligned}
$$

where $a=\left[h_{1}\left(z_{1}+v_{1}\right), h_{2}\left(z_{2}+v_{2}\right)\right]^{\prime}=\left(a_{1}, a_{2}\right)^{\prime}$. 
Proof Given in the appendix.

Corollary 2 For $h_{1}, h_{2}>0$

$$
\begin{aligned}
2^{-2} u\left(x_{1}, x_{2}\right) & +\sum_{j=0}^{\infty}\left(\left[\prod_{k=1}^{2}(-1)^{j_{k}} \delta\left(k, j_{k}\right)\right] F\left(x_{1}, x_{2}\right)\right) \\
& =\frac{-2}{(2 \pi)^{2}} h_{1} h_{2}\left[\sum_{v_{1}=0}^{\infty} \sum_{v_{2}=-\infty}^{\infty} \operatorname{Re}\left[\frac{\phi\left(b_{1}, b_{2}\right) e^{-i x^{\prime} b}}{b_{1} b_{2}}\right]\right]
\end{aligned}
$$

where $b=\left(h_{1}\left(v_{1}+\frac{1}{2}\right), h_{2}\left(v_{2}+\frac{1}{2}\right)\right)^{\prime}=\left(b_{1}, b_{2}\right)^{\prime}$.

The induced integration error is at most

$$
\max \left(\left[1-F\left(x_{1}+\frac{2 \pi}{h_{1}}\right)\right]+\left[1-F\left(x_{2}+\frac{2 \pi}{h_{2}}\right)\right], F\left(x_{1}-\frac{2 \pi}{h_{1}}\right)+F\left(x_{2}-\frac{2 \pi}{h_{2}}\right)\right)
$$

Thus, we can use the bounds employed in Section 2 to provide a way of selecting the step sizes, $h_{1}$ and $h_{2}$.

Although this integration error looks daunting, it is in fact in a very convenient form. This is because to evaluate $F\left(x_{1}, x_{2}\right)$ we would usually have to numerically compute $F\left(x_{1}\right)$ and $F\left(x_{2}\right)$, which means $h_{1}$ and $h_{2}$ have already been chosen by the univariate routines. These selected values will ensure that the error in the bivariate numerical integral is small.

\section{BIVARIATE IMHOF PROCEDURE}

Let us suppose that we are interested in the joint distribution of the two quadratic forms in form variables $y_{1}=u^{\prime} A u$ and $y_{2}=u^{\prime} B u$ where $A$ and $B$ are $T \times T$ matrices and, for sake of simplicity, $u \sim N(0, I)$. The joint characteristic function of $y_{1}$ and $y_{2}$ is given by

$$
\begin{aligned}
\phi\left(t_{1}, t_{2}\right) & =E \exp \left[i \cdot u^{\prime}\left(t_{1} A+t_{2} B\right) u\right] \\
& =\prod_{j=1}^{T}\left(1-2 i \lambda_{j}\left(t_{1}, t_{2}\right)\right)^{-1 / 2},
\end{aligned}
$$

where $\lambda_{j}\left(t_{1}, t_{2}\right)$ denotes the $j$ th eigenvalue of the $\left(t_{1} A+t_{2} B\right)$ matrix. We will be able to use the analytic structure of the characteristic function to rewrite the righthand side of (1) in a productive way. Using the arguments of Imhof (1961) we have

$$
\varepsilon(t)=\arg \left[\phi(t) e^{-i x^{\prime} t}\right]=\left[\left(\frac{1}{2_{j}} \sum_{j=1}^{T} \arctan \left[2 \lambda_{j}\left(t_{1}, t_{2}\right)\right]\right)-x^{\prime} t\right]
$$


and

$$
\gamma(t)=\left|\phi(t) e^{-i x^{\prime} t}\right|=\prod_{j=1}^{T}\left[1+4 \lambda_{j}^{2}\left(t_{1}, t_{2}\right)\right]^{-1 / 4} .
$$

Thus we see that $\operatorname{Re}\left[\phi\left(b_{1}, b_{2}\right) \exp \left(-i x^{\prime} b\right)\right]=\gamma\left(b_{1}, b_{2}\right) \cdot \cos \left[\varepsilon\left(b_{1}, b_{2}\right)\right]$.

If $A$ and $B$ do not commute the use of these expressions to evaluate $\phi\left(t_{1}, t_{2}\right)$ can be very expensive as eigenvalue calculations will be required each time $t_{1}$ and $t_{2}$ vary. An alternative sometimes available is to use the eigenvalue-free technique suggested by Shively, Ansley and Kohn (1990). This exploits the Kalman filter running in complex arithmetic to efficiently compute $\phi\left(t_{1}, t_{2}\right)$. Another approach has been suggested by Krishnaiah $(1977,1980)$ and Khatri et al. (1977) who proposed the use of asymptotic expansions to approximate the joint distribution function.

\section{THE MULTIVARIATE CASE}

The multivariate inversion formula given in Gurland (1948) and Shephard (1991) shows that

$$
u(x)=\frac{(-2)^{p}}{(2 \pi)^{p}} \int_{0}^{\infty} \ldots \int_{0}^{\infty}\left(\Delta_{t_{1}} \cdot \Delta_{t_{2}} \ldots \Delta_{t_{p}}\left[\frac{\phi(t) e^{-x^{\prime} t}}{i t_{1} \ldots i t_{p}}\right]\right) d t
$$

where

$$
\begin{aligned}
u(x)= & 2^{p} F\left(x_{1}, \ldots, x_{p}\right)-2^{p-1}\left[F\left(x_{2}, \ldots, x_{p}\right)+\cdots+F\left(x_{1}, \ldots, x_{p-2}, x_{p-1}\right)\right] \\
& +2^{p-2}\left[F\left(x_{3}, x_{4}, \ldots, x_{p}\right)+\cdots+F\left(x_{1}, \ldots, x_{p-3}, x_{p-2}\right)\right]+\cdots+(-1)^{p} .
\end{aligned}
$$

Theorem 2 now generalises in a straightforward way to deal with $p$ variables. The resulting expression is not very compact and so we have not presented it here. Instead we give the formula corresponding to $z$ being a half as this is gives a useful integration rule. Again we use the expression $\sum_{j=0}^{\infty}$ to stand for the sum over the variables $j_{1}, \ldots, j_{p}$, where these indexes vary between zero and infinity, but never all equal zero simultaneously. Corollary 3 is the result.

Corollary 3 For $h_{1}, h_{2}, \ldots, h_{p}>0$

$$
\begin{aligned}
2^{-p} u(x)+\sum_{j=0}^{\infty}\left(\left[\prod_{k=1}^{p}(-1)^{j_{k}} \delta\left(k, j_{k}\right)\right] F(x)\right) \\
=\frac{(-i)^{p} 2}{(2 \pi)^{p}} h_{1} \ldots h_{p}\left[\sum_{v_{1}=0}^{\infty} \sum_{v_{2}=-\infty}^{\infty} \cdots \sum_{v_{p}=-\infty}^{\infty} \operatorname{Re}\left[\frac{\phi(b) e^{-i x^{\prime} b}}{b_{1} b_{2} \ldots b_{p}}\right]\right],
\end{aligned}
$$


if $p$ is even, and

$$
=-\frac{(-i)^{p-1} 2}{(2 \pi)^{p}} h_{1} \ldots h_{p}\left[\sum_{v_{1}=0}^{\infty} \sum_{v_{2}=-\infty}^{\infty} \cdots \sum_{v_{p}=-\infty}^{\infty} \operatorname{Im}\left[\frac{\phi(b) e^{-i x^{\prime} b}}{b_{1} b_{2} \ldots b_{p}}\right]\right] \text {, if } p \text { is odd }
$$

where $b=\left(h_{1}\left(\frac{1}{2}+v_{1}\right), h_{2}\left(\frac{1}{2}+v_{2}\right), \ldots, h_{p}\left(\frac{1}{2}+v_{p}\right)\right)^{\prime}=\left(b_{1}, b_{2}, \ldots, b_{p}\right)^{\prime}$.

\section{EXAMPLES OF THE USE OF THE BIVARIATE PROCEDURE}

Consider the joint distribution function of the first two non-circular serial correlation coefficients. We follow Durbin's (1980) definition of $r_{j}=y^{\prime} A_{j} y / y^{\prime} y$ for $j=1,2$, as the serial correlation coefficients, where $A_{1}$ and $A_{2}$ are given below

$$
\text { 2. } A_{1}=\left[\begin{array}{ccc}
110 & \cdots & 0 \\
101 & \cdots & 0 \\
010 & \cdots & 0 \\
\vdots \vdots & & \vdots \\
000 & & 11
\end{array}\right] \text { and } A_{2}=2 \cdot A_{1}^{2}-I, \text { as } \cos 2 \theta=2 \cos ^{2} \theta-1
$$

For the sake of simplicity we allow $y$ to be a $T \times 1$ vector, containing independent and identically distributed zero mean Gaussian variables.

The eigenvalues of $A_{j}$ are $\mu_{r_{j}}=\cos [j \pi(r-1) / T],(r=1, \ldots, T)$, while they share the same eigenvectors (see Anderson (1971, pp. 282-290)). This means that we can write

$$
\operatorname{Pr}\left(r_{1} \leqq d_{1}, r_{2} \leqq d_{2}\right)=\operatorname{Pr}\left[\sum_{t=1}^{T} u_{t}^{2}\left(\mu_{r_{1}}-d_{1}\right) \leqq 0, \sum_{t=1}^{T} u_{t}^{2}\left(\mu_{r_{2}}-d_{2}\right) \leqq 0\right]
$$

where $u_{\mathrm{t}} \sim N I D(0,1)$.

Various joint probabilities for the serial correlation coefficients are given in Table 1. In brackets beneath these probabilities are the number of terms in the two dimensional sum used in their computation. The marginal distribution functions were evaluated using Davies (1980) algorithm, with the corresponding induced error chosen to be negligible.

A simulation experiment was performed to check the accuracy of these calculations. When $T$ was less than 100 , one million replications were used in the experiment, while for larger $T$ we slowly reduced the number of replications. The Gaussian white noise variables were generated using the Numerical Algorithms Group (1984) routines G05DDF and G05CBF. The experiment showed that the calculations were accurate to four decimal places in every case.

We can see that there is a surprisingly large probability of observing all the serial correlation coefficients to be positive, and a smaller but non-negligible 
Table 1 Exact joint probability for the first two serial correlation coefficients with $d=1.96 / \sqrt{T}$

\begin{tabular}{cllll}
\hline$T$ & $\operatorname{Pr}\left(X_{1}>d, X_{2} \leqq-d\right)$ & $\operatorname{Pr}\left(X_{1} \leqq-d, X_{2}>d\right)$ & $\operatorname{Pr}\left(X_{1}>d, X_{2}>d\right)$ & $\operatorname{Pr}\left(X_{1} \leqq-d, X_{2} \leqq-d\right)$ \\
\hline 8 & 0.00000 & 0.00001 & 0.00446 & 0.00000 \\
& $(5209)$ & $(5829)$ & $(5822)$ & $(5350)$ \\
16 & 0.00000 & 0.00007 & 0.00389 & 0.00000 \\
& $(1856)$ & $(1338)$ & $(1684)$ & $(1565)$ \\
32 & 0.00000 & 0.00100 & 0.00279 & 0.00000 \\
& $(601)$ & $(664)$ & $(640)$ & $(686)$ \\
64 & 0.00007 & 0.00107 & 0.00204 & 0.00004 \\
& $(373)$ & $(356)$ & $(374)$ & $(358)$ \\
128 & 0.00020 & 0.00101 & 0.00157 & 0.00014 \\
& $(253)$ & $(261)$ & $(234)$ & $(236)$ \\
256 & 0.00031 & 0.00092 & 0.00125 & 0.00023 \\
& $(209)$ & $(214)$ & $(214)$ & $(211)$ \\
512 & 0.00040 & 0.00085 & 0.00105 & 0.00033 \\
& $(201)$ & $(181)$ & $(199)$ & $(203)$ \\
1024 & 0.00047 & 0.00079 & 0.00092 & 0.00041 \\
& $(248)$ & $(246)$ & $(246)$ & $(248)$ \\
2048 & 0.00054 & 0.00077 & 0.00085 & 0.00049 \\
& $(434)$ & $(432)$ & $(440)$ & $(434)$ \\
& & & & \\
$\infty$ & 0.00062 & 0.00062 & 0.00062 & 0.00062 \\
\hline
\end{tabular}

Note: The asymptotic result is calculated using large sample theory.

probability that they alternate in sign-starting with $r_{1}$ being negative. This is rather worrying as these patterns are usually taken to indicate the presence of an AR(1) process.

Three other examples of the use of the bivariate inversion theorem exist. The first is by Shively (1988) in tackling a problem in time series econometrics and the second is by Shephard (1990a) in testing for linear restrictions in two different regression models. Finally, by using this technique Shephard (1990b) derives the distribution function of the maximum likelihood estimator of a noninvertible first order moving average.

\section{CONCLUSION}

In this paper we have developed Riemann sum rules for the inversion of characteristic functions in order to evaluate the distribution function. Although the use of Riemann sums is at first sight primitive, the selection of this technique allows the automatic choice of step sizes. In practice this means that the user only has to be concerned with the issue of truncating the infinite sums at a sensible point. As a result, the suggested rules should be easy to apply in many cases.

In practice these techniques will be used when the characteristic function is cheap to evaluate and the dimension of the integration is reasonably small (less 
than five). In these cases there are possibilities of large computational economies when compared with simulation experimentation techniques.

\section{References}

Anderson, T. W. (1971). The Statistical Analysis of Time Series. John Wiley, New York.

Bohmann, H. (1961). Approximate Fourier analysis of distribution functions. Arkiv för Matematik 4, 99-157.

Davies, R. B. (1973). Numerical inversion of a characteristic function. Biometrika 60, 415-417.

Davies, R. B. (1980). AS 155: The distribution of a linear combination of $\chi^{2}$ random variables. Applied Statistics 29, 323-333.

Durbin, J. (1980). Approximations for densities of sufficient statistics. Biometrika 67, 311-333.

Gurland, J. (1948). Inversion formula for the distribution of ratios. Annals of Mathematical Statistics 19 , 228-237.

Imhof, J. P. (1961). Computing the distribution of quadratic forms in normal variables. Biometrika 48 , $419-426$.

Khatri, C. G., Krishnaiah, P. R. and Sen, P. K. (1977). A note on the joint distribution of correlated quadratic forms. J. Statistical Planning and Inference 1, 299-307.

Krishnaiah, P. R. (1977). On generalised multivariate gamma type distributions and their applications in reliability. In: Proc. Conf. Theory and Appl. of Reliability with Emphasis on Bayesian and NonParametric Methods. (eds. I. N. Shimi and C. P. Tsokos). Academic Press.

Krishnaiah, P. R. (1980). Computations of some multivariate distributions. In: Handbook of Statistics, Vol. 1. (ed. P. R. Krishnaiah). North-Holland Publishing Company, pp. 745-971.

Numerical Algorithms Group (1984). Fortran Library, Mark 11. NAG, Oxford.

Shively, T. S. (1988). An analysis of tests for regression coefficient stability. Journal of Econometrics 39, 367-386.

Shively, T. S., Ansley, C. F. and Kohn, R. (1990). Fast evaluation of the distribution of the DurbinWatson and other invariant test statistics in regression. Journal of the American Statistical Association 85, 676-685.

Shephard, N. G. (1990a). Tabulation of Farebrother's test for linear restrictions in linear regression models under heteroscedasticity. Unpublished paper, Dept. of Statistics, LSE.

Shephard, N. G. (1990b). Evaluating the distribution functions of the maximum likelihood estimators of the first order moving average and the local level model. Unpublished paper, Dept. of Statistics, LSE.

Shephard, N. G. (1991). From characteristic function to distribution function: A simple framework for the theory. Economic Theory (forthcoming).

Zygmund, A. (1955). Trigonometrical Series. Dover, New York.

\section{MATHEMATICAL APPENDIX}

\section{Proof of Theorem 1}

$$
\operatorname{Pr}(X \leqq x-t)-\operatorname{Pr}(X>x+t)=-\frac{2}{2 \pi} \int_{0}^{\infty} \cos [y t]\left(\Delta_{y}\left[\frac{\phi(y) e^{-i y x}}{y i}\right]\right) d y .
$$

Thus

$$
\sum_{j=0}^{\infty} \cos [2 \pi z j][\delta(1, j) \operatorname{Pr}(X \leqq x)]=F(x)+\sum_{j=1}^{\infty} \cos [2 \pi z j][\delta(1, j) \operatorname{Pr}(X \leqq x)]
$$


NUMERICAL INTEGRATION RULES

45

$$
=\frac{1}{2}-\frac{1}{2 \pi} \sum_{j=-\infty}^{\infty} \exp [-2 \pi i j z] \int_{0}^{\infty} \cos [2 \pi j y]\left(\Delta_{y}\left[\frac{\phi\left(h_{1} y\right) e^{-i x h_{1} y}}{i y}\right]\right) d y
$$

Using Proposition 1

$$
\begin{aligned}
= & \frac{1}{2}-\frac{1}{2} \frac{1}{2 \pi} \sum_{v=-\infty}^{\infty}\left(\Delta_{z}\left[\frac{\phi\left(h_{1}(z+v)\right) \exp \left[-i x h_{1}(z+v)\right]}{(z+v) i}\right]\right) \\
= & \frac{1}{2}-\frac{1}{2} \frac{1}{2 \pi} \cdot \Delta_{z}\left[\frac{\phi\left(h_{1} z\right) \exp \left[-i x h_{1} z\right]}{z i}\right] \\
& -\frac{1}{2} \frac{1}{2 \pi} \sum_{v=1}^{\infty}\left(\Delta_{z} \cdot \Delta_{z+v}\left[\frac{\phi\left(h_{1}(z+v)\right) \exp \left[-i x h_{1}(z+v)\right]}{(z+v) i}\right]\right) \\
= & \frac{1}{2}-\frac{1}{2 \pi} \operatorname{Im}\left[\frac{\phi\left(h_{1} z\right) \exp \left[-i x h_{1} z\right]}{z}\right] \\
& -\frac{1}{2 \pi} \sum_{v=1}^{\infty}\left(\Delta_{z} \operatorname{Im}\left[\frac{\phi\left(h_{1}(z+v)\right) \exp \left[-i x h_{1}(z+v)\right]}{(z+v)}\right]\right) .
\end{aligned}
$$

Proof of Theorem 2

$$
\begin{aligned}
\operatorname{Pr}\left(X_{1} \leqq x_{1}-t_{1}, X_{2} \leqq x_{2}-t_{2}\right)-\operatorname{Pr}\left(X_{1} \leqq x_{1}-t_{1}, X_{2}>x_{2}+t_{2}\right) \\
-\operatorname{Pr}\left(X_{1}>x_{1}+t_{1}, X_{2} \leqq x_{2}-t_{2}\right)+\operatorname{Pr}\left(X_{1}>x_{1}+t_{1}, X_{2}>x_{2}+t_{2}\right) \\
=\frac{(-2)^{2}}{(2 \pi)^{2}} \int_{0}^{\infty} \int_{0}^{\infty}\left(\cos \left[t_{1} y_{1}\right] \cos \left[t_{2} y_{2}\right]\right)\left(\Delta_{y_{1}} \cdot \Delta_{y_{2}}\left[\frac{\phi(y) e^{-i x^{\prime} y}}{i y_{1} i y_{2}}\right]\right) d y_{1} d y_{2} .
\end{aligned}
$$

Hence

$$
\begin{aligned}
2^{-2} u\left(x_{1}, x_{2}\right) & +\sum_{j=0}^{\infty}\left(\cos \left[2 \pi z_{1} j_{1}\right] \cos \left[2 \pi z_{2} j_{2}\right]\right) \delta\left(1, j_{1}\right) \cdot \delta\left(2, j_{2}\right) F\left(x_{1}, x_{2}\right) \\
= & \frac{1}{(2 \pi)^{2}} \sum_{j_{1}=-\infty}^{\infty} \sum_{j_{2}=-\infty}^{\infty} \exp \left[-2 \pi i\left[j_{1} z_{1}+j_{2} z_{2}\right]\right] \\
& \times \int_{0}^{\infty} \int_{0}^{\infty}\left(\cos \left[\frac{2 \pi j_{1}}{h_{1}} \cdot y_{1}\right] \cdot \cos \left[\frac{2 \pi j_{2}}{h_{2}} \cdot y_{2}\right]\right)
\end{aligned}
$$




$$
\times\left(\Delta_{y_{1}} \cdot \Delta_{y_{2}}\left[\frac{\phi\left(h_{1} y_{1}, h_{2} y_{2}\right) e^{-i x^{\prime}\left(h_{1} y_{1}, h_{2} y_{2}\right)^{\prime}}}{i h_{1} y_{1} \cdot i h_{2} y_{2}}\right]\right) d y_{1} \cdot d y_{2}
$$

So, using Proposition 1, we have for $p=2$

$$
\begin{aligned}
= & \frac{1}{(2 \pi)^{2}} \cdot \frac{1}{(2)^{2}} h_{1} h_{2} \sum_{v_{1}=-\infty}^{\infty} \sum_{v_{2}=-\infty}^{\infty}\left(\Delta_{z_{1}} \cdot \Delta_{z_{2}}\left[\frac{\phi(a) e^{-i x^{\prime} a}}{i a_{1} i a_{2}}\right]\right) \\
= & \frac{1}{(2 \pi)^{2}} \cdot \frac{1}{(2)^{2}} h_{1} h_{2}\left[\sum_{v_{2}=-\infty}^{\infty}\left(\Delta_{z_{2}} \cdot \Delta_{z_{1}}\left[\frac{\phi\left(h_{1} z_{1}, a_{2}\right) e^{-i x^{\prime}\left(h_{1} z_{1}, a_{2}\right)^{\prime}}}{i h_{1} z_{1} i a_{2}}\right]\right)\right. \\
& \left.+\sum_{v_{1}=1}^{\infty} \sum_{v_{2}=-\infty}^{\infty}\left(\Delta_{z_{1}} \cdot \Delta_{z_{2}} \cdot \Delta_{a_{1}}\left[\frac{\phi(a) e^{-i x^{\prime} a}}{i a_{1} i a_{2}}\right]\right)\right]
\end{aligned}
$$

Thus

$$
\begin{aligned}
2^{-2} u\left(x_{1}, x_{2}\right)+ & \sum_{j=0}^{\infty}\left(\cos \left[2 \pi z_{1} j_{1}\right] \cos \left[2 \pi z_{2} j_{2}\right]\right) \delta\left(1, j_{1}\right) \cdot \delta\left(2, j_{2}\right) F\left(x_{1}, x_{2}\right) \\
= & \frac{-1 / 2}{(2 \pi)^{2}} \cdot h_{1} \cdot h_{2} \cdot\left[\sum_{v_{2}=-\infty}^{\infty}\left(\Delta_{z_{2}} \operatorname{Re}\left[\frac{\phi\left(h_{1} z_{1}, a_{2}\right) e^{-i x^{\prime}\left(h_{1 z_{1}, a_{2} \prime^{\prime}}\right.}}{h_{1} z_{1} a_{2}}\right]\right)\right. \\
& \left.+\sum_{v_{1}=1}^{\infty} \sum_{v_{2}=-\infty}^{\infty}\left(\Delta_{z_{1}} \cdot \Delta_{z_{2}} \operatorname{Re}\left[\frac{\phi(a) e^{-i x^{\prime} a}}{a_{1} a_{2}}\right]\right)\right]
\end{aligned}
$$

Proposition 1 Assume $\Delta_{z_{1}} \cdots \Delta_{z_{p}} g\left(z_{1}, \ldots, z_{p}\right) \in L^{p}$ and is both continuous and bounded. Then

$$
\begin{aligned}
& \sum_{v_{1}=-\infty}^{\infty} \cdots \sum_{v_{p}=-\infty}^{\infty}\left(\Delta_{z_{1}} \cdots \Delta_{z_{p}} g\left(v_{1}+z_{1}, \ldots, v_{p}+z_{p}\right)\right)=2^{p} \sum_{n_{1}=-\infty}^{\infty} \cdots \sum_{n_{p}=-\infty}^{\infty} e^{-2 \pi i n^{\prime} z} \int_{0}^{\infty} \cdots \int_{0}^{\infty} \\
& \quad \times\left(\prod_{j=1}^{p} \cos \left[2 \pi n_{j} y_{j}\right]\right)\left(\Delta_{y_{1}} \cdots \Delta_{y_{p}} g\left(y_{1}, \ldots y_{p}\right)\right) d y_{1} \ldots d y_{p}
\end{aligned}
$$

where $n=\left(n_{1}, \ldots, n_{p}\right)^{\prime}$ and $z=\left(z_{1}, \ldots, z_{p}\right)^{\prime}$.

Proof of Proposition 1 Trivial variant of Poisson's formula, cf. Bohmann (1961) or Zygmund (1955, p. 37). 